\title{
CÂNCER DE PÊNIS NOS HOMENS BRASILEIROS ENTRE OS ANOS DE 2000 E A ATUALIDADE
}

\author{
PENIS CANCER IN BRAZILIAN MEN BETWEEN 2000 AND CURRENT
}

\author{
Lucas Galvão Araújo ${ }^{1}$ \\ Ana Flávia Henriques Ribeiro Monteiro ${ }^{2}$ \\ Ana Paula Monteiro do Nascimento ${ }^{3}$ \\ Mateus Louis Rodrigues Cavalcante ${ }^{4}$ \\ Paloma Medeiros Gomes Cavalcanti ${ }^{5}$ \\ Raissa Cavalcanti Rodrigues ${ }^{6}$
}

RESUMO: Objetivo: Descrever um perfil epidemiológico da incidência e mortalidade de homens vítimas de câncer de pênis no Brasil no século XXI. Método: Utilizou-se a base de dados do The Global Cancer Observatory (GCO) e do INCA, além de artigos encontrados no PUBMED e Google Acadêmico para estudo e correlação com os dados pesquisados. Resultados: Através dos achados científicos analisados, o câncer de pênis é o $22^{\circ}$ tipo de câncer que mais acomete a população masculina no Brasil com incidência de $1,8 \%$ no ano de 2018 , tendo sua mortalidade crescente entre os anos de 2000 e 2018 de acordo com o INCA e com a base de dados do GCO. As regiões com maior mortalidade por câncer de pênis foram a região Norte e Nordeste com, respectivamente, $0,1 \%$ e $0,08 \%$ e as regiões com menor mortalidade foram Sudeste e Sul com, respectivamente, 0,04\% e 0,05\% do total de óbitos por câncer. Entre os anos de 2000 e 2017 no Brasil, ainda segundo o INCA, a mortalidade por câncer de pênis foi maior entre a faixa etária entre 60 e 69 anos, com 1.222 óbitos. Conclusão: Apesar de ser considerado raro, o câncer de pênis ainda é causa de morte principalmente em regiões mais pobres; entretanto, este é um dos poucos tipos de câncer que pode ser evitado com diagnóstico precoce e ações de prevenção. Por isso fica evidente que recursos financeiros devem ser destinados a atender, principalmente através de campanhas educativas a população carente de informações sobre a doença.

Descritores: Epidemiologia. Neoplasias Penianas. Brasil.

\footnotetext{
${ }^{1}$ Estudante de Medicina do $8^{\circ}$ período do Centro Universitário de João Pessoa - UNIPE.

${ }^{2}$ Estudante de Medicina do $8^{\circ}$ período do Centro Universitário de João Pessoa - UNIPE.

${ }^{3}$ Estudante de Medicina do $8^{\circ}$ período do Centro Universitário de João Pessoa - UNIPE.

${ }^{4}$ Estudante de Medicina do $11^{\circ}$ período do Centro Universitário de João Pessoa - UNIPE.

${ }^{5}$ Estudante de Medicina do $8^{\circ}$ período do Centro Universitário de João Pessoa - UNIPE.

${ }^{6}$ Estudante de Medicina do $5^{\circ}$ período do Centro Universitário de João Pessoa - UNIPE.
} 
ABSTRACT: Objective: To describe an epidemiological profile of the incidence and mortality of men suffering from penis cancer in Brazil in the 21st century. Method: The database of The Global Cancer Observatory (GCO) and INCA was used, in addition to articles found in PUBMED and Google Scholar for study and correlation with the researched data. Results: Through the scientific findings analyzed, penis cancer is the 22nd type of cancer that most affects the male population in Brazil with an incidence of $1.8 \%$ in 2018, with its mortality increasing between the years 2000 and 2018 from according to INCA and the GCO database. The regions with the highest mortality from penile cancer were the North and Northeast with $0.1 \%$ and $0.08 \%$, respectively, and the regions with the lowest mortality were Southeast and South with, respectively, $0.04 \%$ and $0.05 \%$ of total cancer deaths. Between 2000 and 2017 in Brazil, according to the INCA, mortality from penile cancer was higher among the age group between 60 and 69 years, with 1,222 deaths. Conclusion: Despite being considered rare, cancer of the penis is still a cause of death mainly in more needy regions; however, this is one of the few types of cancer that can be avoided with early diagnosis and preventive actions. For this reason, it is evident that financial resources must be allocated, mainly through educational campaigns to the population in need of information about the disease.

Keywords: Epidemiology. Penile Neoplasms. Brazil. 


\section{INTRODUÇÃO}

No ano de 2018 foi divulgado no site The Global Cancer Observatory (GCO) que os países com maior incidência de câncer no mundo foram China, Estados Unidos, Índia, Japão, Alemanha e Brasil, sendo os tumores que mais acometeram a população mundial de ambos os sexos, em todas as idades, respectivamente pulmão com 2.093.876 (dois milhões noventa e três mil oitocentos e setenta e seis) casos, mama com 2.088.849 (dois milhões oitenta e oito mil oitocentos e quarenta e nove) casos, colorretal com 1.849 .518 (um milhão oitocentos e quarenta e nove quinhentos e dezoito) casos, próstata com 1.276. 106 (um milhão duzentos e setenta e seis mil cento e seis) casos e estômago com 1.033.701 (um milhão trinta e três mil setecentos e um) casos, excetuando pele, tendo na população masculina, maior incidência os cânceres de pulmão com 1.368.524 (um milhão trezentos e sessenta e quatro mil quinhentos e vinte e quatro) casos, próstata com 1.276.106 (um milhão duzentos e setenta e seis mil cento e seis) casos, colorretal com 1.026.215 (um milhão vinte e seis mil duzentos e quinze) casos, estômago com 683.754 (seiscentos e oitenta e três mil setecentos e cinquenta e quatro) casos e fígado com 596.574 (quinhentos e noventa e seis mil quinhentos e setenta e quatro) casos, sendo o câncer de pênis o $24^{\circ}$ (vigésimo quarto) tipo que mais acomete homens no mundo com 34.475 (trinta e quatro mil quatrocentos e setenta e cinco) casos.

No Brasil, os cânceres com maior incidência na população, de ambos os sexos, em todas as idades, em 2018, são respectivamente o câncer de mama com 85.620 (oitenta e cinco mil seiscentos e vinte) casos, próstata com 84.992 (oitenta e quatro mil novecentos e noventa e dois) casos, colorretal com 51.783 (cinquenta e um mil setecentos e oitenta e três) casos, pulmão com 34.511 (trinta e quatro mil quinhentos e onze) casos e tireoide com 21.470 (vinte e um mil quatrocentos e setenta) casos, ainda segundo o GCO.

Já na população masculina brasileira, em 2018, em todas as idades, os cânceres que possuem maior incidência são próstata com 84.992 (oitenta e quatro 
mil novecentos e noventa e dois) casos, colorretal com 24.737 (vinte e quatro mil setecentos e trinta e sete) casos, pulmão com 19.169 (dezenove mil cento e sessenta e nove) casos, estômago com 12.340 (doze mil trezentos e quarenta) casos e bexiga com 9.127 (nove mil cento e vinte e sete) casos, sendo o câncer de pênis o $22^{\circ}$ (vigésimo segundo) tipo de maior incidência nessa população, com 1.840 (mil oitocentos e quarenta) casos.

As principais causas de câncer de pênis são má higiene íntima, presença de fimose e também o excesso de prepúcio, que estão intimamente relacionados à higiene inadequada, já que podem dificultar a exposição da glande e, assim, a correta higienização, predispondo o indivíduo às infecções, infecção por certos subtipos de papilomavírus humano (HPV), em especial o 16 e o 18 que estão presentes em $50 \%$ dos casos e desempenham papel importante na gênese tumoral, tabagismo, relações sexuais desprotegidas, baixo nível de educação e alcoolismo. Contudo, tais causas não são independentes e pelo menos três devem estar associadas para serem consideradas fatores de risco relevantes (WIND et al., 2019, apud DO COUTO et al., 2014; GAO et al., 2016; COELHO et al., 2018).

A respeito do comportamento biológico do carcinoma epidermóide de pênis, ele tende a ser uniforme, podendo ser uma lesão exofítica, superficial ou ulcerada e pode ter caráter invasivo ou não. Metade dos casos ocorre na região da glande e no prepúcio, mas pode acometer a haste peniana e o escroto. A via linfática é a principal via de disseminação do tumor, principalmente para os linfonodos inguinais, pélvicos e periaórticos, apesar do comprometimento visceral ser raro, bem como metástases por via hematogênica. A progressão da doença é lenta e pacientes não tratados geralmente morrem em decorrência de complicações, como infecção inguinal, necrose e erosão dos vasos femorais (NARDOZZA, 2010).

O tratamento é feito após o estadiamento, através da classificação TNM, que avalia o tumor primário, os linfonodos regionais e a presença de metástases à distância. A depender do estadiamento, podemos fazer uso de 5-fluorouracila ou Imiquimode, ambos fármacos tópicos que tem como finalidade inibir o crescimento de células cancerígenas, ou fazer ressecção a laser ou ressecção parcial ou total do epitélio acometido. Pode ser feito também penectomia parcial (preferência por ser mais conservador) ou penectomia total em casos pertinentes e em tumores mais 
avançados. Após o tratamento, tem a linfadenectomia inguinal bilateral eletiva caso aja acometimento linfonodal (Sociedade Brasileira de Oncologia, 2017).

Consideram-se como fatores preditivos de mal prognóstico e, consequentemente, elevada mortalidade câncer-específica o subtipo histológico, a presença de invasão perineural, de invasão linfovascular, a profundidade da invasão e o grau do tumor (Sociedade Brasileira de Oncologia, 2017).

Pode ser prevenido com a melhora dos hábitos de higiene íntima do órgão, realização de circuncisão nos pacientes com fimose, uso de preservativos para diminuir a chance de contágio de doenças sexualmente transmissíveis, como o HPV, realização de autoexame que realizado regularmente pode contribuir para detecção precoce dessa doença, melhorando o prognóstico e a sobrevida dos pacientes (COSTA et al., 2013).

De acordo com o Portal do Instituto Nacional de Câncer José Alencar Gomes da Silva (INCA) a mortalidade por câncer entre os anos de 2000 e 2017 no Brasil, em ambos os sexos, foi em sua maioria devido a cânceres com sítio primário no pulmão, na mama, na próstata, no estômago e no intestino grosso. Enquanto na população masculina, os cinco principais sítios primários com maior mortalidade entre os anos de 2000 e 2017 no Brasil, foram pulmão, próstata, estômago, esôfago, fígado e vias biliares.

No ano de 2018, no Brasil, segundo dados coletados no GCO, os cânceres com maior mortalidade em ambos os sexos são pulmão com 31.856 (trinta e um mil oitocentos e cinquenta e seis) casos, colorretal com 24.482 (vinte e quatro mil quatrocentos e oitenta e dois) casos, mama com 18.442 (dezoito mil quatrocentos e quarenta e dois) casos, próstata com 16.730 (dezesseis mil setecentos e trinta) casos e estômago com 15.796 (quinze mil setecentos e noventa e seis) casos, sendo mais prevalente nos homens a mortalidade por câncer de pulmão com 18.044 (dezoito mil e quarenta e quatro) casos, próstata com 16.730 (dezesseis mil setecentos e trinta) casos, colorretal com 12.031 (doze mil e trinta e um) casos, estômago com 9.944 (nove mil novecentos e quarenta e quatro) casos e esôfago com 7.645 (sete mil seiscentos e quarenta e cinco) casos, respectivamente, sendo o câncer de pênis o $20^{\circ}$ (vigésimo) tipo de câncer com maior mortalidade na população masculina em 2018, somando 507 (quinhentos e sete) casos. 


\section{MÉTODO}

Trata-se de um estudo epidemiológico, quantitativo, retrospectivo, realizado por meio da consulta ao Portal do Instituto Nacional de Câncer José Alencar Gomes da Silva (INCA), alimentado pelo Ministério da Saúde e na base de dados do site The Global Cancer Observatory (GCO), que utiliza dados de vários projetos-chave da Seção de Vigilância do Câncer (CSU) da IARC, incluindo a GLOBOCAN; Incidência de Câncer nos Cinco Continentes (Cl5); Incidência Internacional de Câncer na Infância (IICC); e Cancer Survival na África, Ásia, Caribe e América Central (SurvCan).

Na pesquisa, foram coletados dados do Portal do INCA dos anos de 2000 a 2017, referentes a mortalidade por câncer de pênis, no Brasil e nas regiões Centrooeste, Nordeste, Norte, Sudeste e Sul. Também foram coletados dados sobre a mortalidade por câncer de pênis por faixa etária da população mundial e brasileira de 2000, entre os anos de 2000 e 2017. Assim como referentes a mortalidade por câncer de pênis por idade, pelas populações mundial e brasileira de 2000, no Brasil e nos estados do Acre, Alagoas, Amapá, Amazonas, Bahia, Ceará, Distrito Federal, Espírito Santo, Goiás, Maranhão, Mato Grosso, Mato Grosso do Sul, Minas Gerais, Paraná, Paraíba, Pará, Pernambuco, Piauí, Rio Grande do Norte, Rio Grande do Sul, Rio de Janeiro, Rondônia, Roraima, Santa Catarina, Sergipe, São Paulo e Tocantins.

No GCO, foram coletados dados do ano de 2018 no mundo e no Brasil sobre incidência e mortalidade de todos os tipos de câncer, em ambos os sexos, em todas as idades, assim como incidência e mortalidade do câncer de pênis, no ano de 2018 em homens de todas as idades no mundo de acordo com os Continentes, Áreas do mundo, Países, Níveis de renda e Índice de Desenvolvimento Humano (IDH), como também o número estimado de casos de câncer de pênis no mundo e no Brasil nos anos de 2020 e 2040. 
Além disso, houve pesquisa bibliográfica em artigos para estudo e correlação com os dados pesquisados nas bases de dados PUBMED e Google Acadêmico.

\section{RESULTADOS E DISCUSSÕES}

No mundo, no sexo masculino, em todas as idades, no ano de 2018 , segundo o GCO, o continente com maior incidência de câncer de pênis é a Ásia com 18.954 (dezoito mil novecentos e cinquenta e quatro) casos, seguido da América com 6.740 (seis mil setecentos e quarenta) casos, Europa com 6.324 (seis mil trezentos e vinte e quatro) casos, África com 2.279 (dois mil duzentos e setenta e nove) casos e Oceania com 178 (cento e setenta e oito) casos, sendo o Brasil responsável pela incidência de aproximadamente $27 \%$ dos casos da América.

Em relação a mortalidade do câncer de pênis, ainda segundo o GCO, no ano de 2018, os continentes seguiram a mesma ordem de incidência, sendo a Ásia o continente com maior mortalidade, com 10.565 (dez mil quinhentos e sessenta e cinco) casos, seguido da América com 1.836 (mil oitocentos e trinta e seis) casos sendo 1.103 (mil cento e três) desses casos na América do Sul, Europa com 1.823 (mil oitocentos e vinte e três) casos, África com 881 (oitocentos e oitenta e um) casos e Oceania com 33 (trinta e três) casos, sendo o Brasil responsável por cerca de $28 \%$ dos casos de mortalidade da América. Além disso, segundo o GCO, a maior incidência e mortalidade no ano de 2018 , foi na população mundial de baixa renda econômica média, porém, em relação ao Índice de Desenvolvimento Humano (IDH), teve maior incidência e mortalidade nos países com maior IDH.

Em 2018, no mundo, de acordo com GCO, a incidência de câncer de pênis era de 34.475 (trinta e quatro mil quatrocentos e setenta e cinco mil), tendo uma perspectiva que em 2020 esse número chegue a 36.230 (trinta e seis mil duzentos e trinta), e em 2040 a 56.519 (cinquenta e seis mil quinhentos e dezenove) número de casos. No Brasil, em 2018, esse número era de 1.840 (mil oitocentos e quarenta), tendo uma perspectiva de aumentar para 1.949 (mil novecentos e quarenta e nove) 
número de casos em 2020 e 3.482 (três mil quatrocentos e oitenta e dois) casos em 2040.

No Brasil, o câncer de pênis é o $22^{\circ}$ tipo de câncer que mais acomete sua população masculina com incidência de 1,8\% no ano de 2018 e tendo sua mortalidade crescente entre os anos de 2000 e 2017 de acordo com o Portal do Instituto Nacional de Câncer José Alencar Gomes da Silva (INCA) e com a base de dados do site The Global Cancer Observatory (GCO). Entre os anos de 2000 e 2003, as mortes por câncer de pênis representaram 0,04\% das mortes do país, subindo para $0,05 \%$ no ano de 2004 , baixando novamente para $0,04 \%$ no ano de 2005 , voltando a subir para 0,05\% de 2006 a 2009, subindo para 0,06\% em 2010, baixando para 0,05\% em 2011 e mantendo-se 0,06\% de 2012 a 2017 segundo o INCA.

Mapa 1: Taxas de incidência de câncer de pênis estimadas no mundo em 2018 padronizadas por idade.

Estimated age-standardized incidence rates (World) in 2018, penis, males, all ages

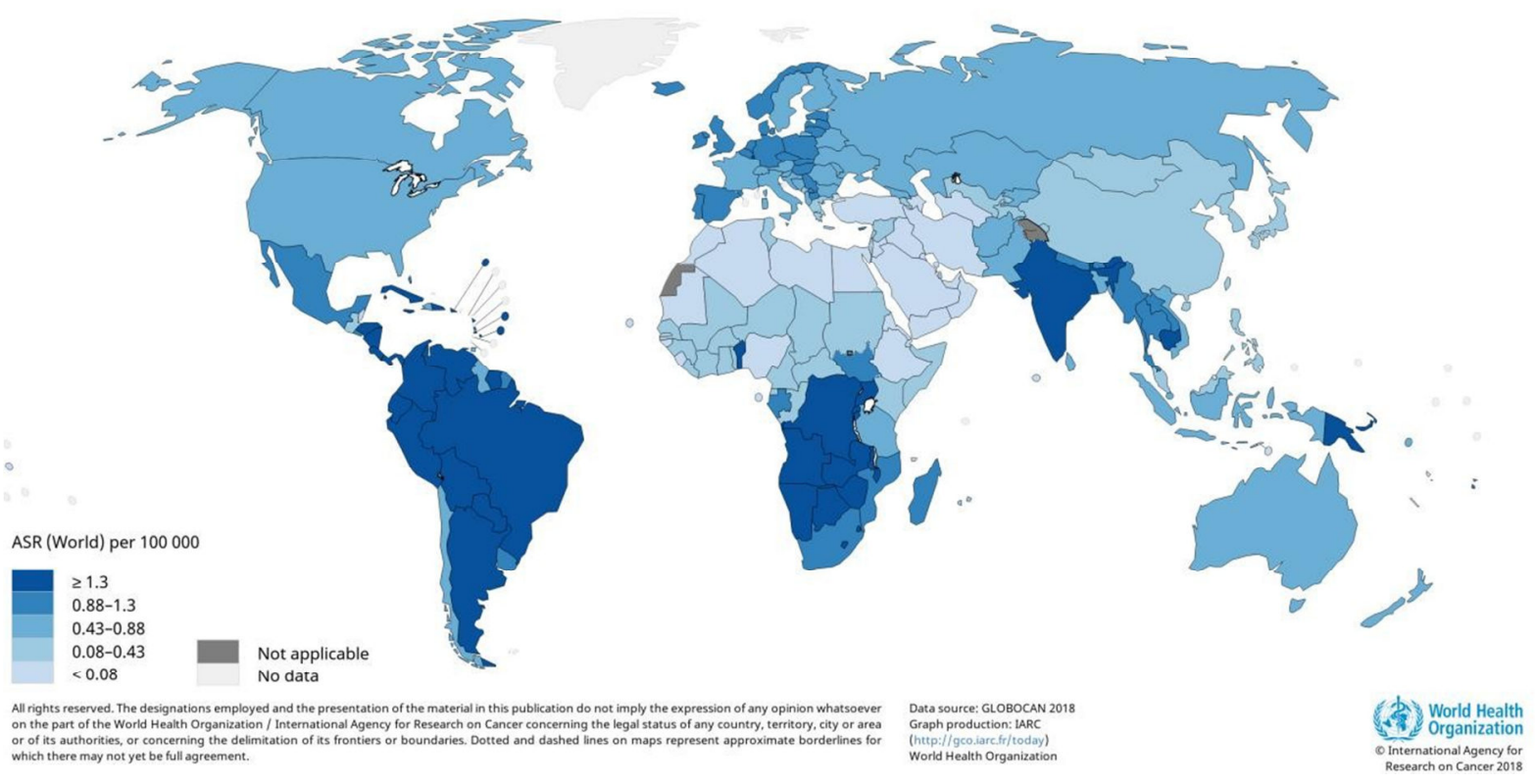

Fonte: The Global Cancer Observatory.

Entre as regiões, no período de 2000 a 2017, a mortalidade por câncer em relação a mortalidade do país variou. Na região Centro-Oeste essa variação ficou entre $0,03 \%$ e $0,08 \%$ da mortalidade do país, sendo 2003 o ano com menor 
mortalidade e 2015 o maior. Os dados mais recentes de acordo com o Atlas de Mortalidade do INCA mostraram uma mortalidade de $0,07 \%$ na região no ano de 2017. No Nordeste, a variação foi menor, $0,04 \%$ a $0,08 \%$, mas apresentou maior mortalidade em mais anos: 2008, 2010, 2012 e mais recentemente em 2017. O período compreendido entre 2000 e 2003 foi quando apresentou menor mortalidade na região. A região Norte exibiu a maior variação dentre as regiões, $0,02 \%$ a $0,1 \%$ durante esses 17 anos. 2003 foi o ano com menor mortalidade e em 2014, 2016 e 2017, maior. As regiões Sul e Sudeste tiveram a mesma variação, 0,03\% a 0,05\%, tendo sido registrada menor mortalidade nos anos de 2001, 2011 e 2012 no Sul e maior em 2007, 2010 e entre 2013 e 2017, enquanto na região Sudeste a menor foi em 2001 e maior em 2013 e 2015.

Entre os anos de 2000 e 2017 no Brasil, ainda segundo o INCA, a mortalidade por câncer de pênis entre 0-4 anos foi de 1 (um) óbito por 100.000 homens (cem mil), de 5-9 anos foi de 0 (zero), de 10-14 anos foi 1 (um), 15-19 anos foi de 4 (quatro), 20-29 anos foi de 82 (oitenta e dois), 30-39 anos foi de 402 (quatrocentos e dois), 40-49 anos foi de 836 (oitocentos e trinta e seis), 50-59 anos foi de 1.171 (mil cento e setenta e um), 60-69 anos foi de 1.222 (mil duzentos e vinte e dois), 70-79 anos foi de 1.037 (mil e trinta e sete) 80 anos ou mais foi de 937 (novecentos e trinta e sete), somando um total de 5.700 mortes por câncer de pênis no Brasil entre os anos de 2000 e 2017.

Segundo Nardozza et al. (2010), o carcinoma epidermóide de pênis é mais prevalente na sexta década de vida, sendo pouco encontrado em jovens e raramente na infância, tal informação acompanha os dados de mortalidade pesquisados no INCA, pois é observado que antes dos 20 anos apenas 6 pessoas no Brasil faleceram pela doença durante esse período de 2000 a 2017 e que também a partir da faixa etária de 30-39 anos temos um aumento progressivo e expressivo do número de óbitos, que só decresce a partir dos 70-79 anos.

$\mathrm{Na}$ representação espacial do Brasil, os estados com maior taxa bruta de mortalidade por câncer de pênis, por 100.000 homens, entre os anos de 2000 e 2017 foram Piauí, com 0,62, Tocantins, com 0,54, e Maranhão, com 0,49; e os estados com menor taxa de mortalidade: Amapá, com 0,19, Acre e Roraima, ambos com 0,22, e São Paulo, com 0,25. 
Mapa 2: Representação espacial das taxas brutas de mortalidade por câncer de pênis, por 100.000 homens, pelas unidades da federação do Brasil, entre 2000 e 2017.

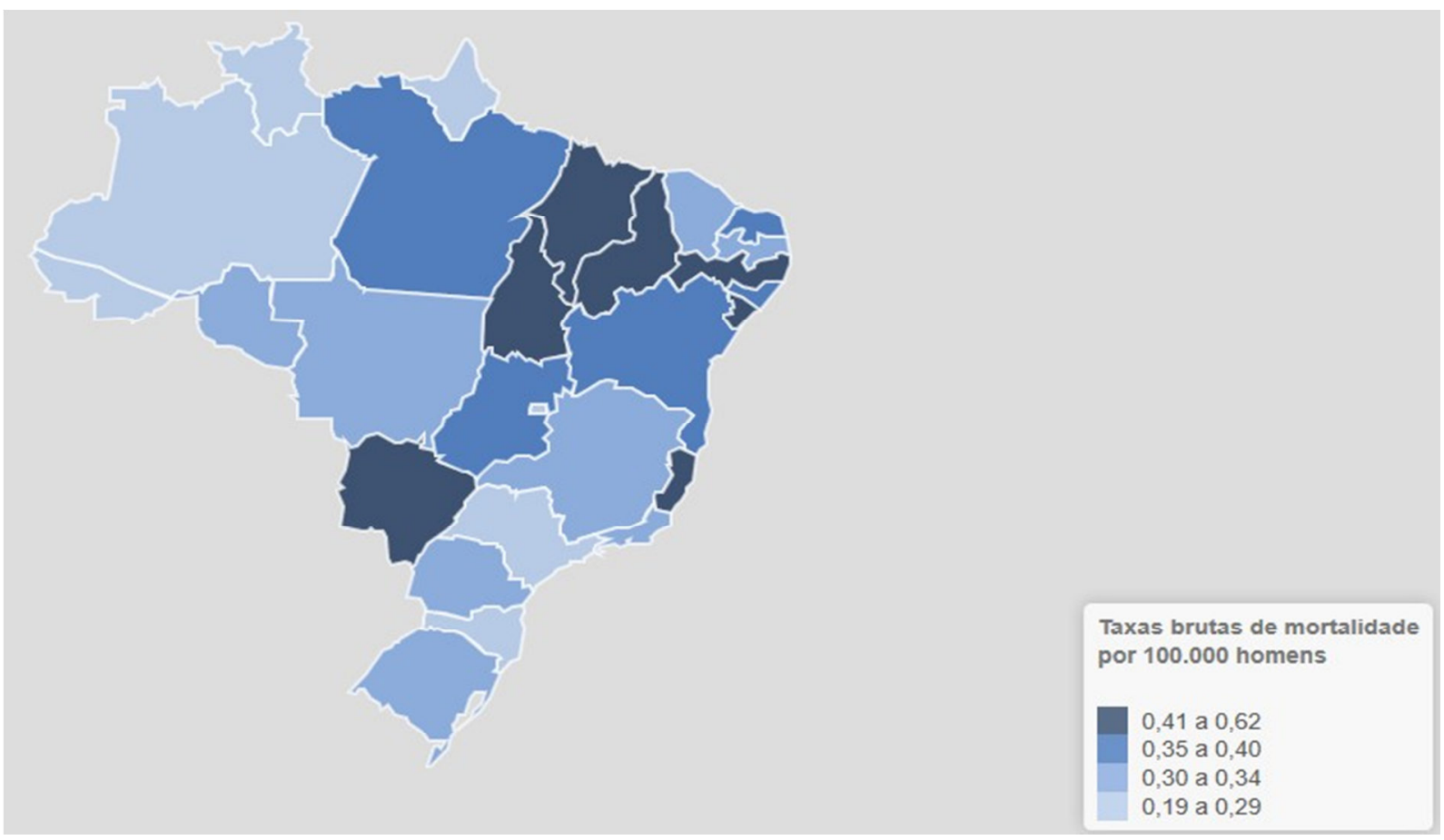

Fonte: Atlas de mortalidade por câncer no Brasil - INCA.

De acordo com Nardozza et al. (2010), na década de 80, nas regiões Norte e Nordeste do Brasil, o carcinoma epidermóide de pênis (CEP) representava 16\% dos tumores malignos em homens adultos e era a terceira neoplasia mais frequente do trato geniturinário, perdendo apenas para os cânceres de próstata e bexiga. Como foi visto nos resultados da pesquisa, as regiões Norte e Nordeste ainda trazem consigo as maiores taxas de mortalidade do país e isso se deve, principalmente, ao desenvolvimento socioeconômico local e também revela fragilidades de acesso ao sistema de saúde e de medidas socioeducativas voltadas para prevenção desse tipo de neoplasia (SANTANA, 2018).

Acredita-se que o aumento do número de mortes por câncer de pênis no Brasil se deve principalmente ao crescimento da população e a inversão da pirâmide etária no país. Mesmo a mortalidade variando diferentemente entre as regiões no decorrer dessas quase duas décadas, os números mais recentes mostram também 
uma ampliação do acesso da população masculina aos serviços de saúde e melhora do rastreio em regiões mais desfavorecidas, ainda que muito ainda precise ser feito tendo em vista que nessas regiões muitas vezes a causa de morte é atrelada a complicações que surgiram por causa do câncer e não diretamente a ele, gerando um estado de subnotificação da doença.

\section{CONCLUSÃO}

Apesar de ser considerado um tumor raro se comparado com outros cânceres mais incidentes em nosso país, o câncer de pênis ainda é causa de morte no Brasil, além de ter forte impacto psicossocial e no bem-estar do homem, principalmente pelo fato do órgão estar relacionado com a forma como ele se enxerga e se define e por muitas vezes, na tentativa de tratar a doença, optar-se por tratamentos mais agressivos, como a penectomia total ou parcial. Entretanto, este é um dos poucos tipos de câncer que podem ser evitados, por isso a importância do diagnóstico precoce e de campanhas de prevenção nesses casos. Quando prevenido ou diagnosticado nos estágios iniciais, as chances de cura são maiores e há aumento da sobrevida do paciente.

Por esse motivo fica evidente que os recursos financeiros devem ser destinados a atender, através o de campanhas educativas a população carente de informações sobre esta doença, como por exemplo, sobre a realização de autoexame, a melhora dos hábitos de higiene íntima, sobre o que é fimose e a postectomia, sobre a importância do uso de preservativos para diminuir a chance de adquirir HPV e outras doenças sexualmente transmissíveis, tudo a fim de contribuir para detecção precoce dessa patologia, melhorando o prognóstico.

Isto posto, deve ser incentivado a realização de pesquisas que avaliem a ocorrência de tumores menos prevalentes, mas que ainda possuem impacto na nossa sociedade em diversas áreas geográficas, como é o caso do câncer de pênis. Os índices de incidência bruta, apesar de possuírem algumas limitações, podem fornecer informações úteis, principalmente no sentido de monitorar a frequência de 
determinada doença ao longo do tempo e permitir avaliar a efetividade de medidas preventivas aplicadas.

Assim, com a intenção de garantir a integralidade do cuidado que se busca na política de saúde pública deve-se fortalecer por meio da construção de redes para promoção de saúde a iniciativa de ir até onde os homens estão além de incentivar mais estudos do tema.

\section{REFERÊNCIAS BIBLIOGRÁFICAS}

Ministério da Saúde, Instituto Nacional de Câncer. Atlas de mortalidade por câncer no Brasil: 2000 a 2017. INCA, Rio de Janeiro. 2020

COSTA, Syvoney et al. CÂNCER DE PÊNIS: EPIDEMIOLOGIA E ESTRATÉGIAS DE PREVENÇÃO. Cadernos de Graduação: Ciências Biológicas e da Saúde Facipe, Recife, v. 1, n. 2, p.23-33, nov. 2013.Disponível em: $<$ https://periodicos.set.edu.br/index.php/facipesaude/article/view/1197>. Acesso em: 07 jan. 2020.

NARDOZZA JÚNIOR, A; ZERATTI FILHO, M; REIS, B R. Urologia fundamental. São Paulo: Planmark, 2010.

SANTANA, Lucas Costa de. Câncer de pênis e o papel da vacinação de meninos contra o HPV na sua prevenção: uma revisão bibliográfica. 2018. 47 f. Monografia (Graduação em Medicina) - Universidade Federal de Sergipe, Lagarto, 2018.

WIND, Mariana Malagoni et al. Câncer de pênis: aspectos epidemiológicos, psicológicos e fatores de risco. Brazilian Journal Of Development. Curitiba, p. 14613-14623. set. 2019. Disponivel em: <http://www.brjd.com.br/index.php/BRJD/article/view/3137>. Acesso em: 07 jan. 2020.

Sociedade Brasileira de Oncologia. Câncer de Pênis: Doença local, loco regional e metastática. 2017.2 Disponível em: $<$ <ttps://www.sboc.org.br/images/diretrizes/diretrizes_pdfs/Cancer_de_penis_vf_2017.pdf>. Acesso em: 07 jan. 2020. 